Relations industrielles

Industrial Relations

\title{
Bélanger, Paul R., Michel Grant et Benoît Lévesque, sous la direction de, La modernisation sociale des entreprises
}

\section{Jean Boivin}

Volume 50, numéro 1, 1995

URI : https://id.erudit.org/iderudit/051002ar

DOI : https://doi.org/10.7202/051002ar

Aller au sommaire du numéro

Éditeur(s)

Département des relations industrielles de l'Université Laval

ISSN

0034-379X (imprimé)

1703-8138 (numérique)

Découvrir la revue

Citer ce compte rendu

Boivin, J. (1995). Compte rendu de [Bélanger, Paul R., Michel Grant et Benoît

Lévesque, sous la direction de, La modernisation sociale des entreprises].

Relations industrielles / Industrial Relations, 50(1), 231-233.

https://doi.org/10.7202/051002ar

Tous droits réservés @ Département des relations industrielles de l'Université Laval, 1995
Ce document est protégé par la loi sur le droit d'auteur. L’utilisation des services d’Érudit (y compris la reproduction) est assujettie à sa politique d'utilisation que vous pouvez consulter en ligne.

https://apropos.erudit.org/fr/usagers/politique-dutilisation/ 
recent years as job classification systems have been made more flexible. However, the specific firm focus remains. The alternative perspective and one that is reflected in some of the papers in this volume is on employability. Many workers will continue to have career jobs but many will not, and will not know their own prospects in advance. The best long run policy to promote employment security appears to be one in which workers are adapt- able and can more easily find alternative employment in the case of job dislocation. This perspective puts more emphasis on the system of education and training and less on employment protection policies intended to preserve a given distribution of jobs and to limit the extent of change in the labour market.

DOUGLAS A. SMITH Carleton University

\section{La modernisation sociale des entreprises}

sous la direction de Paul R. BÉlANGER, Michel GRANT et Benoît LÉVESQUE, Montréal, Les Presses de l'Université de Montréal, 1994, 320 p., ISBN 2-7606$1633-9$.

Ce volume est un collectif d'auteurs comprenant treize textes répartis en trois sections et précédés d'une introduction signée par les coéditeurs.

Le choix du titre est fort pertinent pour décrire le contenu de l'ouvrage et il s'avère d'emblée supérieur aux expressions utilisées dans la littérature portant sur les relations industrielles ou sur les sciences de l'administration et que l'on désigne habituellement par « innovations dans l'organisation du travail " ou encore par " nouvelles stratégies dans la gestion des ressources humaines". En effet, le terme "modernisation sociale " rêfère à la fois aux processus d'ajustements qui sont en train de s'opérer dans les entreprises, dans le contexte d'un environnement plus concurrentiel et qui revêtent des aspects technologiques, organisationnels, institutionnels et culturels et à la fois aux tentatives d'introduire plus de démocratie au travail par une plus grande implication des travailleurs dans les décisions qui les concernent.

La première section comporte quatre textes qui fournissent des explications théoriques aux transformations actuelles. Les deux premiers s'inspirent de l'approche de la régulation (néo-marxiste), le troisième de celle des choix stratégi- ques (Kochan, Katz, McKersie) et le quatrième fait une brève synthèse de la littérature sur la culture d'entreprise. Cette diversité épistémologique rend bien compte des enjeux sur lesquels porterait, selon les codirecteurs du volume, le nouveau modèle d'entreprise émergent. D'un côté, on retrouverait le modèle patronal idéal en vertu duquel on mise uniquement sur la réorganisation du travail conjuguée à une forte identification à l'entreprise et où on ne voit pas la nécessité d'entretenir des relations patronales-syndicales puisque le conflit est considéré comme inexistant. De l'autre, il y aurait le modèle syndical idéal, où tout serait négociable dans le cadre de mécanismes conjoints et paritaires.

Ces deux modèles sont proposés par les codirecteurs comme des prototypes idéaux extrêmes qui permettent "de situer le jeu des acteurs sociaux et de rendre compte de l'instabilité de certaines configurations d'entreprises, tout en renversant le discours le plus courant sur les résistances aux changements attribuées aux syndicats et aux travailleurs " (p. 10).

Même si les codirecteurs affirment que ce cadre d'analyse ou cette typologie permet de situer les sept études de 
cas que l'on retrouve dans la deuxième section, nous avons plusieurs réserves vis-à-vis cette prétention. D'abord, les auteurs et auteures de ces monographies n'ont pas effectué leurs travaux en ayant à l'esprit un tel cadre d'analyse et encore moins ont-ils ou ont-elles cherché à utiliser l'un ou l'autre des trois approches théoriques mentionnées dans la première, à l'exception évidemment de celles qui ont été réalisées par les codirecteurs eux-mêmes. Nous ne sommes pas ici en présence d'un projet collectif où toutes les études particulières ont été effectuées à partir d'un même devis théorique, comme ce fut le cas par exemple pour l'ouvrage de Chaykowski et Verma intitulé Industrial Relations in Canadian Industries (1992).

Ces commentaires n'enlèvent cependant rien à la valeur et à l'utilité des études de cas dont chacune contribue à jeter un regard pertinent sur les transformations qui ont présentement cours dans les entreprises.

Les deux premières monographies sont celles qui collent le plus au modèle de participation négociée suggéré par les codirecteurs dans l'introduction. Cela va de soi, car elles ont été réalisées chacune par un des codirecteurs. Le premier cas est celui d'une usine de fabrication de meubles située à Rockport et l'autre concerne l'usine GM à Boisbriand. Dans les deux cas, on constate que le modèle de participation et d'implication mis en place a été fortement influencé par les résistances initiales du syndicat pour finalement déboucher sur un compromis acceptable (et fort efficace du point de vue des résultats organisationnels) par les deux parties.

La troisième étude de cas porte sur l'impact de l'implantation de systèmes intégrés d'information dans trois usines différentes. Cet impact est évalué à la fois sur les contremaîtres et sur les responsables de la planification de la production. Dans les trois usines con- cernées par cette étude, le rôle des contremaîtres a été profondément modifié, ceux-ci devant dorénavant consacrer moins de temps aux aspects techniques et davantage aux aspects de gestion et de transmission d'informations que par le passé. Quant aux planificateurs, ils voient une partie de leurs tâches et responsabilités traditionnelles leur échapper au profit de l'ordinateur. Dans les deux cas, on assiste à des tensions et à des résistances de la part des intéressés, bien que le nouveau système semble donner des résultats satisfaisants pour les entreprises. Ceux et celles qui ne peuvent fonctionner dans un tel environnement quittent l'organisation.

Le quatrième texte analyse les nouveaux modes de gestion utilisés dans deux alumineries utilisant des technologies de pointe, qui ont cherché à mobiliser les travailleurs au moyen d'une forte culture d'entreprise tout en évitant la syndicalisation. L'auteur nous apprend que, malgré les prêtentions de la direction, l'organisation du travail dans ces deux usines, loin de dépasser le taylorisme, "accentue le contrôle sur le travail ouvrier niant de la sorte la créativité de celui-ci et le réduisant à une implication minimale" (p. 207).

Le texte suivant sort des cadres de l'entreprise pour se situer au niveau du secteur industriel. Il relate les conditions qui ont entouré la mise en place d'un comité paritaire sur la formation de la main-d'œuvre et la création d'un institut spécialisé dans l'industrie chimique et pétrochimique. Tout en qualifiant cette expérience de très positive puisqu'elle constitue une " réalisation éloquente des nouveaux modes de négociations et de coopération qui se mettent en place au sujet de la formation professionnelle " (p. 220), l'auteure ne va pas jusqu'à conclure que ces nouveaux partenariats peuvent être interprétés comme ouvrant la voie à une concertation sur tous les fronts puisque plusieurs entreprises du même secteur annonçaient des fermetures d'usines et des coupures de postes. 
Celle-ci avance néanmoins l'hypothèse que " la question de la formation professionnelle prendra appui sur un certain partenariat qui pourrait déplacer le terrain des négociations patronales-syndicales " (p. 221).

Les deux dernières études de cas concernent les relations entre l'entreprise et les communautés locales dans lesquelles celle-ci s'insère. Dans un premier temps, on relate l'expérience de la mise en opération de l'usine Papiers Cascades de Cabano sous la direction des frères Bernard et Alain Lemaire et avec la participation active de la population locale selon les auteurs. L'implantation de cette usine n'est compréhensible qu'en considérant l'interaction entre l'entrepreneur privé pressenti et divers acteurs locaux comme le conseil municipal, le comité de survivance et les producteurs de bois.

Enfin, la dernière monographie s'intéresse aux PME industrielles de la région de St-Georges de Beauce. Le cadre d'analyse utilisé en est un qui s'inspire des études sur les milieux industriels locaux caractérisés par la présence de nombreuses PME. Celui-ci fait ressortir l'importance des rapports de coopération à côté des rapports de concurrence dans les relations économiques interentreprises. Les auteurs identifient ainsi trois règles sociales, dites " régionales " ou "supra-régionales " qui permettent d'assurer la coordination de l'activité économique autrement que par le marché et par la hiérarchie (p. 254): la priorité de soutien à la PME locale; l'entraide économique, que ce soit sous forme d'actionnariat, de regroupement d'entreprises ou de sauvetage d'entreprises en difficulté ; ainsi qu'une règle de relations industrielles, la proximité sociale travailleurs-employeurs.

Enfin, la dernière section comporte deux textes qui montrent l'évolution du discours de la CSN et de la FTQ face à la réorganisation du travail et aux nouvelles stratégies de gestion des ressources humaines adoptées par les entreprises. Il s'agit là de deux documents fort pertinents et qui arrivent à point pour expliquer les transformations radicales survenues à l'intérieur de ces centrales au cours des dernières années. Le seul reproche que l'on puisse leur adresser est que ces deux textes ne tiennent pas compte des prises de position les plus récentes adoptées par ces centrales (1991 pour la CSN et 1993 pour la FTQ). Dans le cas de la CSN, l'omission est moins importante, car déjà les auteurs ont bien présenté la direction du changement qui était en train de s'opérer. Pour ce qui est de la FTQ, il est regrettable que l'essentiel de la réflexion porte sur un congrès spécial tenu par cette centrale en 1987 sur les nouvelles stratégies patronales ainsi que sur la période antérieure à 1990. Les auteurs auraient sans doute nuancé davantage leurs propos s'ils avaient pu tenir compte des positions adoptées par la FTQ à son congrès de 1993.

Bref, en conclusion, un ouvrage fort intéressant, qui arrive à point pour aider à comprendre les transformations importantes qui sont en train de s'opérer dans les entreprises québécoises et dont je recommande fortement la lecture aux personnes intéressées par le sujet.

JEAN BOIVIN Université Laval 